\title{
ELECTROMAGNETIC FIELD COMPUTATION IN LINEAR ELECTROMAGNETIC ACTUATORS USING THE MESHLESS LOCAL PETROV GALERKIN METHOD
}

\author{
Nadia BENBOUZA*, Fatima Zohra LOUAI*, Mouloud FELIACHI**, Abdelhalim. ZAOUI*** \\ ${ }^{*}$ LSPIE Laboratory, Batna-2- University, Chahid M ${ }^{\text {ed }}$ El Hadj Boukhlouf Street, 05000 - Batna - Algeria, \\ E-mail: b_nadia_dz@yahoo.fr, FZ_Louai@yahoo.com \\ **37 University Boulevard, BP 406, 44602 Saint Nazaire, France, E-mail: mouloud.feliachi@univ-nantes.fr \\ ${ }^{* * *}$ BP17 Bordj El Bahri 16100 Alger, Algiers, Algeria, E-mail: zaoui_abdelhalim@yahoo.fr
}

\begin{abstract}
Meshless methods have attracted considerable interest to the flexibility of adding or removing nodes in the domain of study without any need to remeshing with elements. In this paper the meshless local Petrov Galerkin (MLPG) method is applied to electromagnetic field with a moving structure. The method is an effective truly meshless method for solving PDEs. Formulations are based on a local weak form using the moving least square (MLS) for interpolation schemes. This paper focuses on the application of this method to electromagnetic linear actuators which are finding increasing use in various fields. The device presents an axisymmetric structure with a moving part displaced longitudinally with a constant step. The global magnetic force was calculated using the meshless magnetic potential solutions. Numerical results were compared to the FEM ones considering the structure both in pause and movement cases.
\end{abstract}

Keywords: Moving least square (MLS) approximation, Weight and Test functions, Domain of influence, MLPG method, Actuator, Movement

\section{INTRODUCTION}

Although the mesh-based finite element method is dominant in engineering now, it faces difficulties in solving problems involving large deformation and discontinuities [1]. In recent years, strong interest is focused on the development of the next generation of computational methods called meshless or meshfree methods [2]. The main objective of these ones is to get grid off, or at least to alleviate the difficulty of meshing and remeshing the entire structure; by only adding or deleting nodes in the entire domain of study [3, 4]. The meshless approach does not need a mesh generation to divide the problem domain, the necessary information to provide a solution, is only the location of scattered nodes in the region and on the boundary [2]. Several meshless approaches have been developed, based on global weak forms and which may be classified as Galerkin methods. These methods use a background mesh for the numerical integration of the weak form which may also become distorted during large deformations; therefore these methods are not truly meshless [1-6]. Recently, methods based on the local weak forms have been proposed, the meshless local Petrov-Galerkin method based on a local weighted residual method [Atluri and Zhu (1998)] has attracted a lot of attention and extended too many problems [1-8]. In the MLPG method, both the trial function and test functions are constructed on local subdomains, and only local background integration cells is required. The choice of the test and trial functions is very flexible [4], here; the MLS weight function is used as test function in the local weak form. The shape function constructed by the interpolation, does not possess the Kroneker delta property, the penalty approach is used to enforce the essential boundary conditions [2-4].
In the present work, an MLPG algorithm is implemented for 2D magnetodynamic field problems and applied to an electromagnetic linear actuator device. The study began by considering the plunger in a static position as a magnetostatic problem. Then the study aims to evaluate the variation of global magnetic force in function of the displacement of the moving part, using only some nodes in the computation. Every new position is considered as a new magnetostatic problem. The movement is taken into account by only modifying the physical properties of released or occupied regions [9] or by adding and deleting some nodes associated to the moving band.

Meshless results are in good agreement compared to FEM ones by making a judicious choice of some meshless numerical parameters.

\section{WEIGHT FUNCTIONS}

The weight function is an important ingredient in the moving least squares interpolation (MLS) largely cited in the literature and used to form the trial function by generating the shape function [2-8]. The weight function is defined with a compact support, often called domain of influence. This domain can be circular, elliptic or rectangular. Its width is defined by:

$d_{m I}=d_{\max } C_{I}$

where $d_{\max }$ is a scaling factor and $C_{I}$ the difference between node $X_{I}$ and its nearest neighbour when the nodal distribution is uniform. If the nodes are not uniformly distributed $C_{I}$ is taken as follows:

$C_{I}=\max _{J \in S_{J}}\left\|X_{J}-X_{I}\right\|$ 
where $S_{J}$ is the minimum set of neighbouring points of $X_{I}$ which construct a polygon surrounding point $X_{I}$.

Cubic spline, exponential and Gaussian functions are some of the commonly used weights. The weight function used in this paper is the cubic spline function:

$$
w(r)=\left\{\begin{array}{cl}
\frac{2}{3}-4 r^{2}+4 r^{3} & \text { for } r \leq \frac{1}{2} \\
\frac{4}{3}-4 r+4 r^{2}-\frac{4}{3} r^{3} & \text { for } \frac{1}{2}<r \leq 1 \\
0 & \text { for } r>1
\end{array}\right.
$$

where $r=d_{I} / d_{m I}$ is the normalized distance with $d_{I}=\left\|X-X_{I}\right\|$.

In $2 \mathrm{D}$, the influence domain covers an area [6]. In this work, a rectangular shape domain is chosen and the interpolation is calculated using a linear basis function.

\section{AXISYMMETRIC FORMULATIONS}

The electromagnetic equation describing the axisymmetric problem, in terms of the magnetic vector potential $\vec{A}$, is given by:

$$
\overrightarrow{c u r l}\left(\frac{1}{\mu} \overrightarrow{c u r l} \vec{A}\right)+\sigma \frac{\partial \vec{A}}{\partial t}=\vec{J}
$$

where $\mu, \sigma$ et $\vec{J}$ are respectively the magnetic permeability, the electric conductivity and the external current density.

The essential and flux boundary conditions are respectively:

$$
A=A_{0} \text { on } \Gamma_{u} \quad \text { and } \quad \frac{1}{r} \frac{\partial A}{\partial n}=q_{0} \text { on } \Gamma_{q}
$$

where: $\Gamma_{u} \cup \Gamma_{q}=\Gamma$ is the boundary of $\Omega$ and $\mathrm{n}$ is the unit outward direction to the boundary $\Gamma_{q}$.

Using 2D cylindrical $r, z$ coordinates, and as the magnetic vector potential $\vec{A}$ has only the $\varphi$ component ( $\vec{A}=\vec{A}_{\varphi}$ ), the equation (4) became:

$-\frac{\partial}{\partial r}\left(\frac{1}{r} \frac{1}{\mu} \frac{\partial\left(r A_{\varphi}\right)}{\partial r}\right)-\frac{\partial}{\partial z}\left(\frac{1}{\mu} \frac{\partial\left(A_{\varphi}\right)}{\partial z}\right)=J_{\varphi}$

Equation (6) can also be written as

$$
-\frac{\partial}{\partial r}\left(\frac{1}{r} \frac{1}{\mu} \frac{\partial\left(r A_{\varphi}\right)}{\partial r}\right)-\frac{\partial}{\partial z}\left(\frac{1}{r} \frac{1}{\mu} \frac{\partial\left(r A_{\varphi}\right)}{\partial z}\right)=J_{\varphi}
$$

Using the modified vector potential $A=r A_{\varphi}$ leads to:

$$
-\frac{\partial}{\partial r}\left(\frac{1}{r} \frac{1}{\mu_{r}} \frac{\partial A}{\partial r}\right)-\frac{\partial}{\partial z}\left(\frac{1}{r} \frac{1}{\mu_{r}} \frac{\partial A}{\partial z}\right)=\mu_{0} J_{\varphi}
$$

where $\mu_{r}$ is the relative magnetic permeability and $\mu_{0}$ is the permeability of free space.

In a sub-region $\Omega_{s}=\Omega_{\text {te }}$ (of boundary $\partial \Omega_{s}$ ) located entirely inside the global domain, the local weighted residual form with the penalty method used to enforce essential boundary conditions gives [4]:

$$
\begin{aligned}
& \int_{\Omega}\left(-\frac{\partial}{\partial r}\left(\frac{1}{r} \frac{1}{\mu_{r}} \frac{\partial A}{\partial r}\right)-\frac{\partial}{\partial z}\left(\frac{1}{r} \frac{1}{\mu_{r}} \frac{\partial A}{\partial z}\right)-\mu_{0} J_{\varphi}\right) \cdot v d \Omega \\
& +\int_{\Gamma s u}\left(A-A_{0}\right) \cdot v d \Gamma=0
\end{aligned}
$$

where $A$ is the trial function obtained by the MLS interpolant, $v$ is the test function, $\alpha$ is the penalty parameter, $\Gamma_{s}=\partial \Omega_{s} \cap \Gamma, \Gamma_{s u}=\Gamma_{s} \cap \Gamma_{u}$ and $\Gamma_{s q}=\Gamma_{s} \cap \Gamma_{q}$ (see Fig. 1). Using the divergence theorem, the local weak form of governing equation will be:

$$
\begin{aligned}
\int_{\Omega s}\left(-\frac{\partial}{\partial r}\left(\frac{1}{r} \frac{1}{\mu_{r}} \frac{\partial A}{\partial r}\right)\right. & \left.-\frac{\partial}{\partial z}\left(\frac{1}{r} \frac{1}{\mu_{r}} \frac{\partial A}{\partial z}\right)-\mu_{0} J_{\varphi}\right) \cdot v d \Omega \\
+\alpha \int_{\Gamma s u} A v d \Omega & -\int_{\Gamma s u} q v d \Gamma-\int_{L s} q v d \Gamma \\
& -\int_{\Gamma s q} q_{0} v d \Gamma-\alpha \int_{\Gamma s u} A_{0} v d \Gamma=0
\end{aligned}
$$

The test functions can be chosen to vanish on $L_{s}$, therefore the term $[v q]_{L s}$ vanishes.

Note that the MLS approximation of $A$ can be written as:

$$
A(r, z)=\sum_{I=1}^{n} \phi_{I}(r, z) A_{I}
$$

where: $\phi_{I}(r, z)$ is the MLS shape function of node $I$.

$A_{I}$ are the nodal values of $A$ and $\mathrm{n}$ is the number of nodes. Then the weak form leads to the resulting system of equations:

$$
k^{i n t} A+k^{b d r y} A=f^{i n t}+f^{b d r y}
$$

where 'int' and 'bdry' denote internal and boundary nodes respectively:

$$
\begin{aligned}
& k_{i j}^{(i n t)}=\int_{\Omega^{(i)}} \frac{1}{r \mu_{r}}\left(\frac{\partial \phi_{j}}{\partial r} \frac{\partial v_{i}}{\partial r}+\frac{\partial \phi_{j}}{\partial z} \frac{\partial v_{i}}{\partial z}\right) d \Omega \\
& k_{i j}^{(b d r y)}=\alpha \int_{\Gamma s u^{(i)}} \phi_{j} v_{i} d \Gamma-\int_{\Gamma s u^{(i)}} \frac{\partial \phi_{j}}{\partial n} v_{i} d \Gamma
\end{aligned}
$$

$$
f_{i}^{(i n t)}=\int_{\Omega s^{(i)}} \mu_{0} J_{\varphi} v_{i} d \Omega
$$

$f_{i}^{(b d r y)}=\alpha \int_{\Gamma s u^{(i)}} A_{0} v_{i} d \Gamma-\int_{\Gamma s q^{(i)}} q_{0} v_{i} d \Gamma$

Numerical integration of Gauss is used to evaluate the integrals involved in equations (13) to (16) in every subdomain $\Omega_{s}$.

In the present work, the test function is defined in a similar manner as the weight function in the MLS approximation, by replacing the $d m_{i}$ by $d m_{0 i}$ called support domain of node $\mathrm{i}$ :

$d m_{0 i}=d_{\max 0} \cdot C_{i}$ 
The scaling factors are chosen to ensure a symmetric $\Omega_{s}$, so the test functions $v_{i}(X)$ were chosen to vanish on $\Gamma_{s u}^{i}$ such as $v_{i}=0$ on $\Gamma_{s u}^{i}$ for node i.

The implementation of the MLPG method can be carried out according to the flowchart cited in [3].

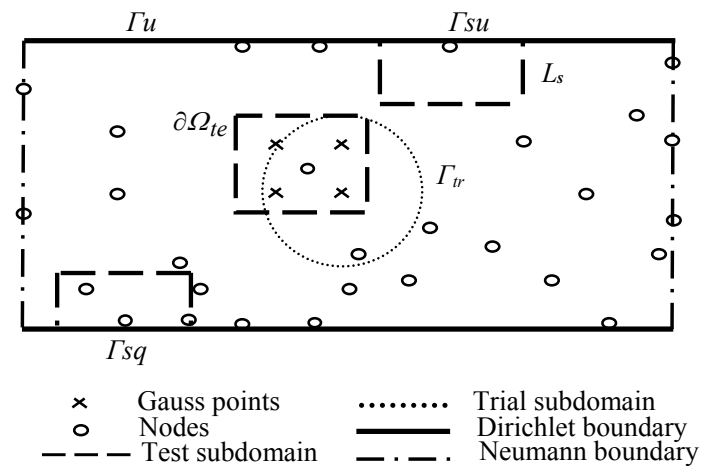

Fig. 1 Principle of MLPG method

Note that in MLPG method, just a cloud of nodes and their corresponding domain of influence are used. The domain of each point contain some nodes in the neibeirhood making a virtual connectivity. No fixed nodal connectivity to form elements as in the universal FEM. Also, no shadow mesh for the domain integrals as in some meshless methods which makes them not trully meshless.

\section{APPLICATIONS AND RESULTS}

The proposed method is applied to an axisymmetric actuator device which represents an electromagnetic hummer with a simple configuration. The system consists of (see Fig. 2 (a)):

1- One cylindrical coil supplied by a constant current impulse $\mathrm{I}=90 \mathrm{~A}$ and containing $N=1178$ of spires.

2- A ferromagnetic non conducting cylindrical plunger $\left(\mu_{r} \approx 230, \sigma=0\right)$ is moving longitudinally towards the coil with a constant step.

3- The free space, containing the air, surrounds the load and the inductor.

The electromagnetic field is pulling the core inside the coil with effect of a magnetic force determined at each step (regular) of the displacement.

The axisymmetric problem structure is presented in Fig. 2 (b), essential boundary conditions are imposed on all sides $\left(\mathrm{A}_{0}=0\right)$.

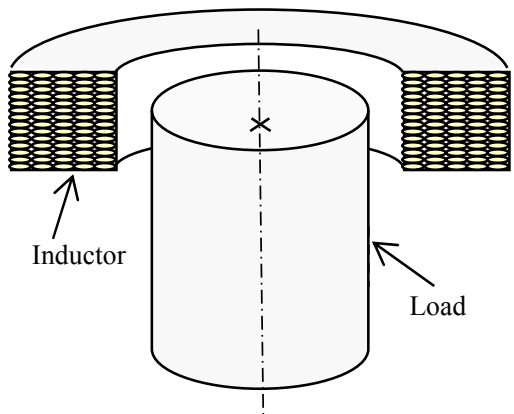

Fig. 2 (a) 2D Representation of the linear actuator

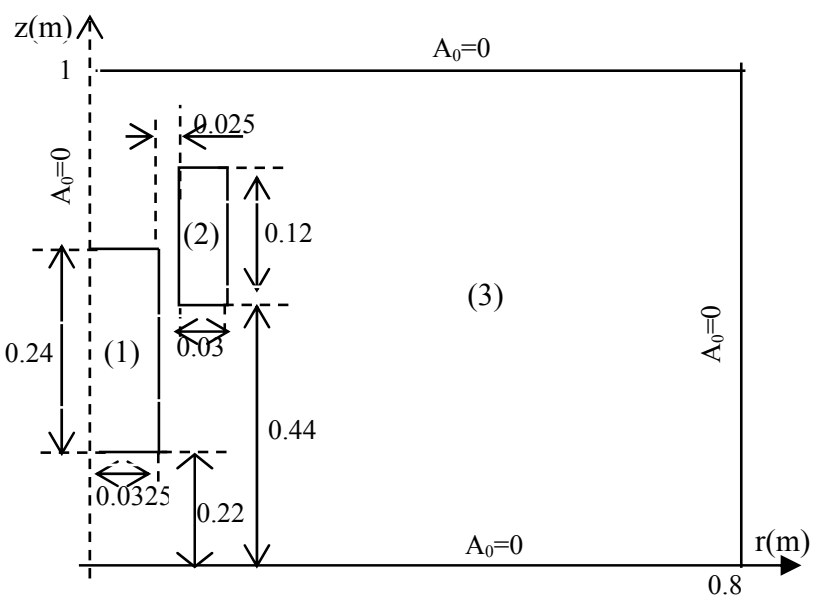

Fig. 2 (b) Axisymmetric problem structure

The dynamic problem is considered as a series of magnetostatic problems, each one for every new position of the load.

The magnetic energy, in axisymmetric coordinate's case, is calculated using the formulation:

$$
W_{\text {mag }}=\pi \int_{\text {coil }} J_{\varphi} A d r d z
$$

Where $A$ is the magnetic vector potential and $J_{\varphi}$ the excitation current density.

Then, the total magnetic force, for a regular increment of displacement $\Delta z$ is calculated as:

$$
F_{\text {mag }}=\frac{d W_{\text {mag }}}{d z}
$$

The realization of all simulations have been achieved in Matlab environment.

\section{A. PLUNGER AT A FIXED POSITION}

A set of $(55 \times 51)$ irregular nodes is generated to represent the problem domain at the initial position $z_{0}=0.22 m$ of the load (Fig. 3).

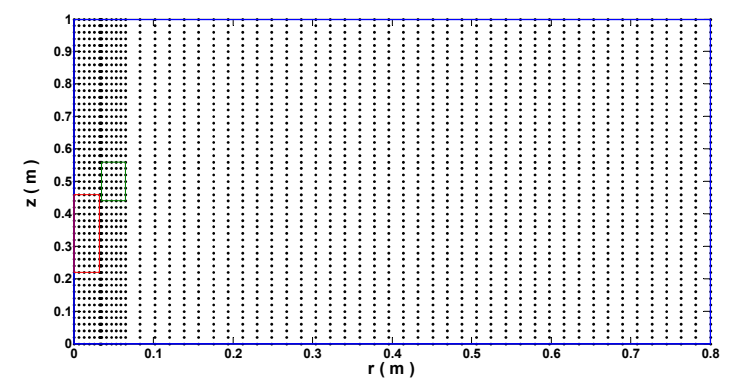

Fig. 3 Nodal arrangement on domain of study and boundaries at depart position $(55 \times 51)$ nodes

The meshless accuracy can be performed by selecting the adequate numerical parameters of the method. All MLPG calculations were achieved by the choice of the following factors: $d_{\max }=1.66, d_{\max 0}=1.35,6 \times 6$ of Gauss points in every node sub-domain $\Omega_{s}$ (without any partition) and 3 points on each section $\Gamma_{s u}$ with the penalty factor of $\alpha=10^{8}$. 
To validate meshless results, these ones are compared to FEM where a large number of nodes (51369 nodes) is used to approximate almost exactly the exact solutions.

Meshless and FEM isovalues contours of the magnetic vector potential, corresponding to the initial position, are shown in Fig. 4 and Fig. 5 respectively.

Fig. 6 shows computed solutions along the line $r=12 \Delta r(\Delta \mathrm{r}$ is the step along $r$ direction) passing through the inductor. As it is noticed, results are in good agreement.

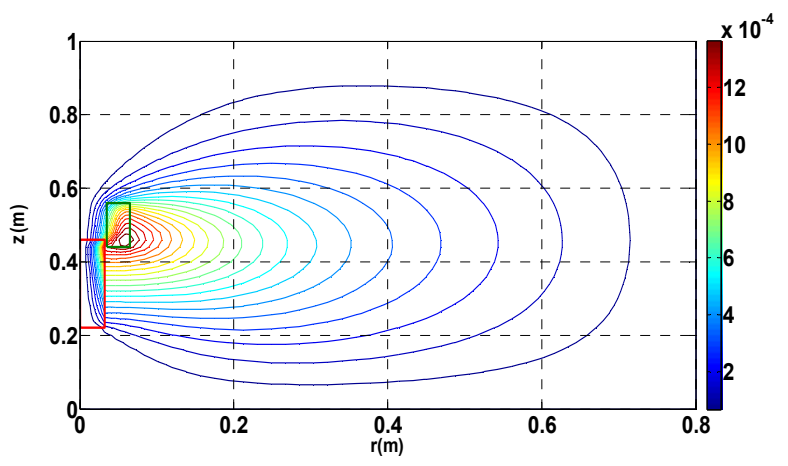

Fig. 4 FEM equipotential lines of the magnetic vector potential $A$

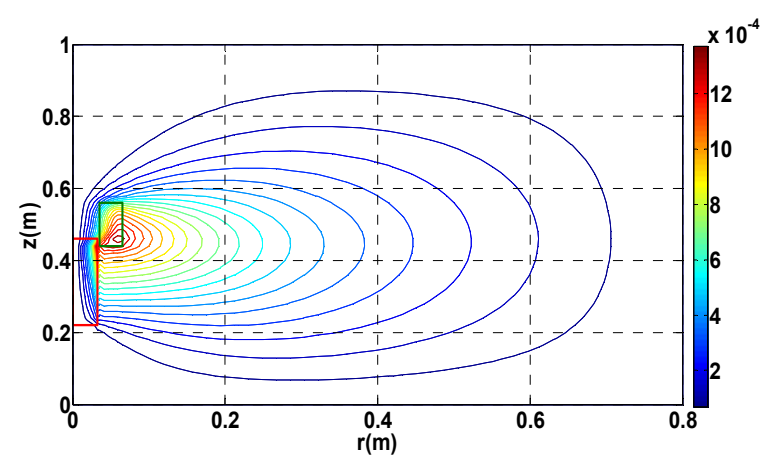

Fig. 5 MLPG equipotential lines of the magnetic vector potential $A$

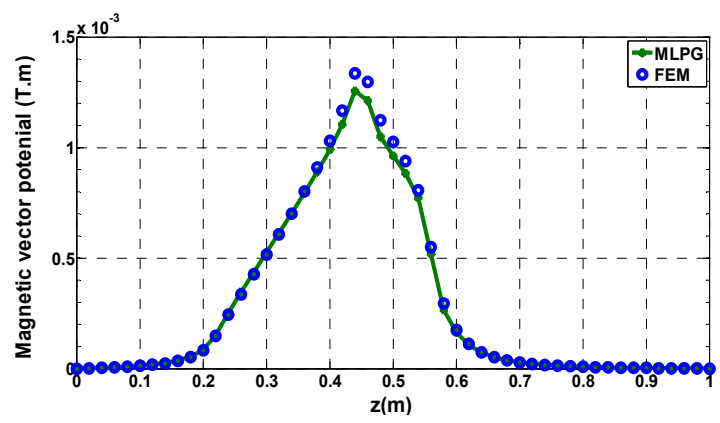

Fig. 6 Meshless and FEM solutions along the line $(12 \Delta r, z)$

\section{B. THE PLUNGER IN MOVEMENT}

\section{First study}

In this case, the displacement step $\Delta z$ is equal or multiple of the regular discretization step $\Delta h$. When the armature moves with an increment $\Delta z$, physical properties assigned to sub-regions released or occupied will be changed according to their new assignment (air or plunger) leading to a new magnetostatic problem. The meshless Magnetic force curve compared with the FEM show a good similitude with the two solutions (Fig. 7). The plunger was displaced over the distance $z=0.2 \mathrm{~m}$ by the step $\Delta z=0.02 m$.

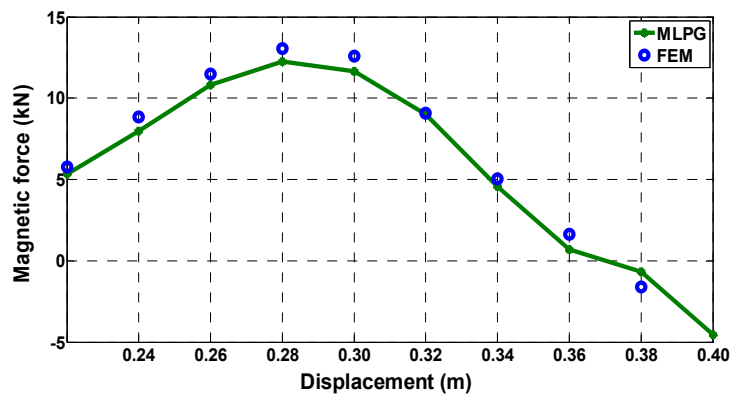

Fig. 7 MLPG and FEM Magnetic forces for $\Delta z=0.02 m$

\section{Second study}

In this case, the displacement step is different from the discretization one. The problem is treated by only moving, adding or deleting nodes in the concerned band of movement as a succession of magnetostatic problems.

The meshless and FEM magnetic forces are calculated for a displacement of $z=0.19 m$ by the step $\Delta z=\Delta h / 2=0.01 \mathrm{~m}$. As it is seen in Fig. 8, the curves are very close.

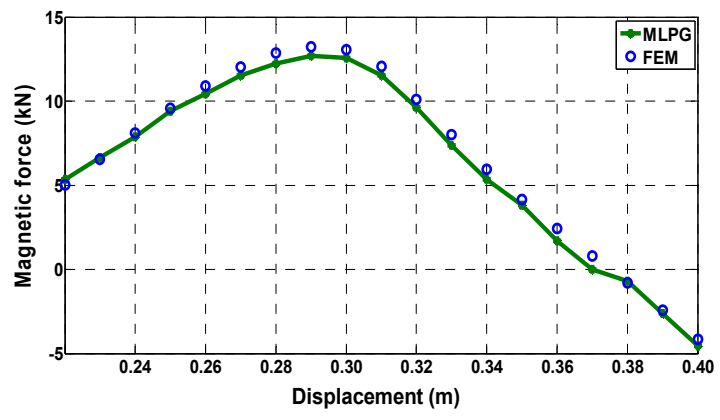

Fig. 8 MLPG and FEM magnetic forces for $\triangle z=0.01 \mathrm{~m}$

\section{CONCLUSION}

The MLPG method is a truly meshless method since no finite elements mesh and no background mesh is required in the interpolation or in the integration in solving a proposed problem. The method is implemented by using the moving least square approximation and the local weak form as well as the penalty technique to enforce the essential boundary conditions.

In the present work, the method was presented to study magnetic field problems and applied to a real device, an axisymmetric linear actuator. The moving part was displaced longitudinally with a constant step.

The study is based only on some nodes in the domain and its boundaries. The displacement of the body is taking in account by only changing the magnetic properties of concerning nodes in the moving band or by adding and deleting some nodes in every new position. This show the flexibility in dealing with the problem of movement. 
The meshless method does not need a new generation of all nodes or the burdensome remeshing with fixed connectivities during the evolution of the simulation, which is the case of the universal FEM.

However, meshless calculations need a judicious choice of some numerical parameters affecting the accuracy: Number of domain nodes and Gauss points, alpha parameter for imposing essential boundary conditions, and finally sizes of the weight and test functions domains. MLPG results, for the selected adequate factors, were compared to the FEM ones showing good agreement between corresponding curves.

\section{REFERENCES}

[1] ZHANG, X. - YAO, Z. - ZHANG, Z.: Application of MLPG in large deformation analysis, Acta Mechanica Sinica, Vol. 22, Issue 4, pp. 331-340, 2006.

[2] IKUNO, S. - TAKAKURA, K. - KAMITANI, A.: Influence of method for imposing essential boundary condition on meshless Galerkin / Petrov-Galerkin approaches, IEEE Trans Magn., Vol. 43, No. 4, pp. 1501-1504, Apr. 2007.

[3] ATLURI, S. N. - ZHU, T.: A new meshless local Petrov-Galerkin (MLPG) approach in computational mechanics, Computational Mechanics, Vol. 22, pp. 117-127, 1998.

[4] TLURI, S. N. - SHEN, S.: The meshless local Petrov-Galerkin (MLPG) method: A simple and lesscostly alternative to the finite element and boundary element methods, CMES: Comp. Modeling Eng Sci, Vol. 3, pp. 11-51, 2002.

[5] FRIES, T. P. - MATTHIES, H. G.: Classification and Overview of mesh-free methods, Department of Mathematics and Computer Science, Technical University Braunschweig, Tech. Rep., [online], 2004.

[6] DOLBOW, J. - BELYTSCHKO, T.: An introduction to programming the Element-free Galerkin method, Archives of Computational Methods in Engineering 05 (1998), pp. 207-241.

[7] ABBASBANDY, S. - SHIRZADI, A.: A Meshless method for two dimensional diffusion equation with an integral condition, Engineering Analysis with Boundary Elements, Vol. 34, No.12, pp. 1031-1037, 2010.

[8] SATAPRAHM, C. - LUADSONG, A.: The meshless local Petrov-Galerkin method for simulating unsteady incompressible fluid flow, Journal of the Egyptian Mathematical Society 22, pp. 501-510, 2014.

[9] SRAIRI, K. - FELIACHI, M.: Electromagnetic actuator behaviour analysis using finite element and parameterization methods, IEEE transactions on magnetics, Vol. 31, No.6, November 1995.

Received February 17, 2016, accepted April 29, 2016

\section{BIOGRAPHIES}

Nadia Benbouza was born in Batna (Algeria) in 1964. She received the engineer degree in electrical engineering from the university of Batna, Algeria in 1990; the magister degree in industrial electricity in 1997. In1998, she joined the same university as assistant professor. Her research interests include electromagnetic and meshless methods.

Fatima-Zohra Louai was born in Algiers (Algeria) in 1967. She received the engineer degree in electrical engineering from the University of Batna, Algeria in 1990, the DEA degree in electrical engineering from l'INPG de Grenoble (France) in 1991 and the doctorate degree from the University of Nantes (France) in 1995. In 1995, she joined the Electrical Engineering Institute, University of Batna (Algeria), as an Assistant Professor. She was promoted to Associate Professor in 1998. Since 2000, she has been head of the "Modelling of Electromagnetic Phenomena" (MEP) team in LSPIE research laboratory. Her research interests include electromagnetic and thermal phenomena, hysteresis modelling and meshless methods.

Mouloud Féliachi was born in Biskra, Algeria. He received the engineer degree in Electronics from "Ecole Nationale Polytechnique" of Algiers in 1976, the engineer doctorate degree from the "Conservatoire National des Arts et Métiers" of Paris in 1981 and the doctorate Sciences degree from "Institut National Polytechnique" of Grenoble in 1986, all in electrical engineering. In 1987, he worked for Leroy Somer Company in Orleans. In 1988, he joined the University of Nantes (Technological Institute of Saint-Nazaire) where he is a Professor. From 1998 to 2000, he served as scientific director of the Inductive Techniques Research Lab (LRTI). From 1993 to 2003, he was head of the Modeling and Simulation team in Electrical Engineering Lab (GE44). He is currently director of the professional "License" degree, in industrial maintenance. His research is being conducted in IREENA $\mathrm{Lab}$, and area of interests hybrid analytical and numerical modelling of electromagnetic phenomena and devices, with emphasis on eddy current non destructive testing and Evaluation.

Abdelhalim Zaoui was born in Labiadh, SidiCheikh (Algeria) in 1969. He received the engineer degree in electrical engineering from ENITA, Algiers, (Algeria) in 1993. In 2008, he received the PHD in electrical engineering from Nantes University (France). He is now a lecturer in the Military Polytechnic School in Algiers, Algeria. His main research activities include the design and computation of electromagnetic devices. 